\title{
Similarities between digits' movements in grasping, touching and pushing
}

\author{
Jeroen B. J. Smeets · Juul Martin · Eli Brenner
}

Received: 31 July 2009/Accepted: 17 March 2010/Published online: 9 April 2010

(C) The Author(s) 2010. This article is published with open access at Springerlink.com

\begin{abstract}
In order to find out whether the movements of single digits are controlled in a special way when grasping, we compared the movements of the digits when grasping an object with their movements in comparable single-digit tasks: pushing or lightly tapping the same object at the same place. The movements of the digits in grasping were very similar to the movements in the single-digit tasks. To determine to what extent the hand transport and grip formation in grasping emerges from a synchronised motion of individual digits, we combined movements of finger and thumb in the single-digit tasks to obtain hypothetical transport and grip components. We found a larger peak grip aperture earlier in the movement for the single-digit tasks. The timing of peak grip aperture depended in the same way on its size for all tasks. Furthermore, the deviations from a straight line of the transport component differed considerably between subjects, but were remarkably similar across tasks. These results support the idea that grasping should be regarded as consisting of moving the digits, rather than transporting the hand and shaping the grip.
\end{abstract}

Keywords Human · Prehension · Grasping · Finger · Thumb

\section{Introduction}

About ten years ago, we hypothesised that grasping is controlled as the independent movements of digits towards

J. B. J. Smeets $(\varangle) \cdot$ J. Martin · E. Brenner

Research Institute MOVE, Faculty of Human Movement

Sciences, VU University Amsterdam, van der Boechorststraat 9,

1081 BT Amsterdam, The Netherlands

e-mail: J.Smeets@fbw.vu.nl positions on an object's surface (Smeets and Brenner 1999). In this view, the curved paths (i.e. the opening and closing of the grip) emerge because the digits tend to approach the surface perpendicularly. We showed that a minimum-jerk model (Flash and Hogan 1985) based on this view is quantitatively consistent with many aspects of grasping without any further assumptions or parameterfitting (Smeets and Brenner 1999). Such a model can deal with responses to perturbation in object position and size (Smeets et al. 2002a), and also explain the time-dependency of the effects of illusions on grip formation (Smeets et al. 2002b; Smeets et al. 2003).

We also provided some experimental support for the independent control of the digits. For instance, grasping with the finger and thumb of a single hand is very similar to grasping with the index fingers of both hands, and in terms of the variability in the movements, the digits are to a large extent independent (Smeets and Brenner 2001). Furthermore, obstacles influence the speed of grasping as predicted if one regards the limitations on the individual digits' paths (Biegstraaten et al. 2003). The fact that the digits' paths during the closing of the grip are influenced by the orientation of the surface (Kleinholdermann et al. 2007) provided direct evidence that the approach of the digits to the object's surface is not a simple closing of the grip, but consists of movements that tend to approach the surface perpendicularly.

Nevertheless, many authors question this view on grasping (Mon-Williams and McIntosh 2000; Bennis and Roby-Brami 2002; Kamper et al. 2003; van de Kamp and Zaal 2007) and continue to use peak grip aperture as a measure for visual size processing (Ganel et al. 2008; Franz et al. 2009). The aim of the present study is to provide a further test of the hypothesis of independent digit control for grasping. To do so, we compare movements of the 
digits in grasping with those in two single-digit tasks. We try to make the task constraints (e.g. start and end position, required precision and contact force) similar for the three tasks. In the present study, we therefore compare the movements of the active digits in grasping with those in two other tasks: lightly tapping (touching) the object from the side and pushing the object sideways. The three tasks were set up so that the active digits had same start and goal positions and more or less the same precision requirements. The main difference is that both digits are active in grasping, whereas only one is active in pointing and touching (we refer to the digit that is not used to perform the task as the passive digit). However, as the constraints are not exactly the same (e.g., the movement of the thumb is also constraining the movement of the index finger in grasping, but not in the single-digit tasks), we cannot expect the movements of the digits to be exactly the same. Therefore, we formulate three predictions that do not rely on an exact match of the task constraints.

An experimental fact that seems to be in conflict with our view is that the movements of the thumb and finger are not the same (or more precisely: they are not each other's mirror image) when grasping. For instance, it has been reported that the path of the thumb is less curved than that of the finger (Wing and Fraser 1983; Paulignan et al. 1997). Such differences between the digits' paths are largely due to non-equivalent contacts points. In natural grasping, contacts positions are generally chosen so that the thumb has to move a shorter distance. But even with equivalent contact positions, there remain differences between the digits (Smeets and Brenner 2001). This does not necessarily argue against independent control. It might be that differences in shape between the digits, or other anatomical properties, lead due to different precision requirements. These differences between finger and thumb are present in any task, independent of the precise constraints. If the hypothesis of independent digit control were correct, one would expect the same deviation from a mirror image in grasping as for the active digits in touching and pushing. This is the first prediction of independent digit control that we will test.

The second prediction is about the most commonly used parameter to describe grasping: peak grip aperture. If the hypothesis of independent digit control is correct, one may expect that the peak grip aperture in grasping and the imaginary peak grip aperture that can be constructed by combining movements of finger and thumb from corresponding single-digit trials should be the same. This will very likely not be exactly true, as the constraints will slightly differ. Despite such differences, we can expect peak grip aperture and its timing to be systematically related: larger peak grip apertures (in more accurate tasks) occur earlier in the movement (Smeets and Brenner 1999).
So if we find differences in peak grip aperture, we will test whether this depends on timing in the same way for all tasks. If such a relationship is found, we can test whether this relationship follows the quantitative predictions of the minimum-jerk model.

The third prediction is about the effects of the independence of the digits on the variability. The first part of this prediction is that the digits that are active in touching, pushing or grasping are controlled in the same way in all tasks. Therefore, the precision of the movements of the active digits will be the same. According to our view, the characteristics of the movements follow from the constraints to movements of the individual digits. As the movement of the passive digit in the single-digit task is much less constrained than that of the active digit, we predict that a digit will be more variable when passive than when active.

\section{Methods}

Subjects

Eight subjects took part in the experiment: one author (EB: S5), one visiting scientist experienced in grasping research (S6), and six colleagues of the VU University who were not involved in grasping research. Five of the subjects were women, and the other three were men; their mean age was $34.4(\mathrm{SD}=9.5)$. All subjects were right-handed and had normal or corrected-to-normal vision; none of them reported any physical injuries or anomalies affecting their performance. Except for the co-author, all subjects were naïve with respect to the specific hypothesis being tested.

\section{Procedure and experimental setup}

The main aim of the setup was to create equal levels of comfort and difficulty for all subjects, while motor demands were equal for thumb and index finger both within and between tasks. A graphic representation of the setup is shown in Fig. 1.

Subjects were asked to sit on a stool that was adjusted in height so that subjects' belly buttons coincided with the edge of the experimental table. The subjects' feet remained in contact with the floor. Subsequently, subjects were asked to stretch their dominant arm and place their hand flat on the table in the median plane. The position of the wrist was marked. Next, subjects were given the experimental cube (sides $5 \mathrm{~cm}$., mass $364 \mathrm{~g}$.) and asked to place it on the marked spot, and orient it in a manner that maximised their comfort. The individual preferred position and orientation of the cube were marked and used during the rest of the experiment. The starting position was $30 \mathrm{~cm}$ from the 


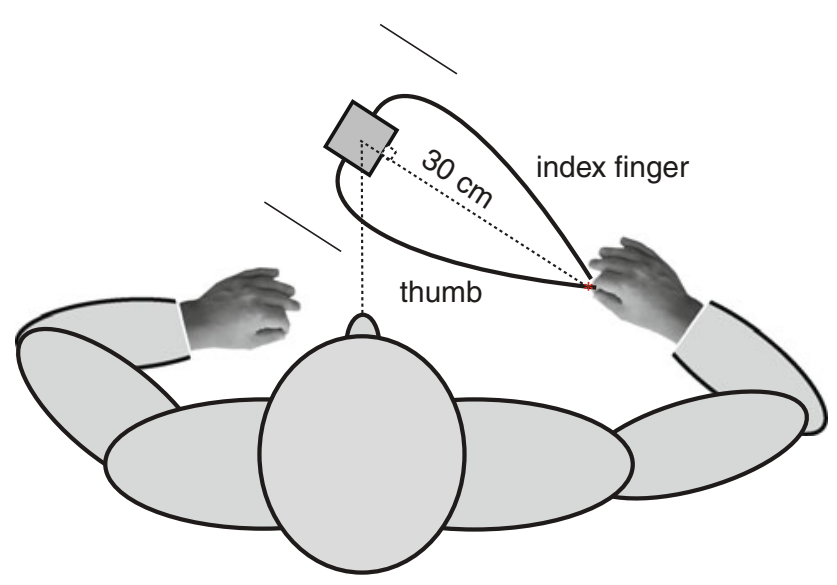

Fig. 1 Top view of the experiment. The two thin lines indicate the position to which the subject had to push the cube. The dashed lines indicate how the cube was aligned to the subject. The thick curves are hypothetical paths of index finger and thumb

cube, perpendicular to the centre of the face of the cube that was closest to the right shoulder. Subjects were asked to hold the tips of their index finger and thumb in contact at this starting position at the beginning of each trial.

Five conditions had to be performed. The instructions were to grasp the object with a precision grip (i.e. with the object between thumb and index finger) and lift it; to touch the object on the side without making it move using the thumb; to touch the object on the side without making it move using the index finger; to push the object away using the thumb; or to push the object away using the index finger. In the latter two conditions, subjects were instructed to move the cube until it passed across a line that was $10 \mathrm{~cm}$ from the far side of the cube. Having to push the cube in a specified direction with one digit made it important to make contact near the centre of the cube's surface. Each condition was performed twenty times: the conditions were randomly intermixed. The instruction was incorporated in the verbal go-signal (for instance: 'push with thumb', 'grasp', or 'touch with finger').

Movements were recorded at $200 \mathrm{~Hz}$ with an Optotrak 3020 camera unit (Northern Digital, Waterloo, Canada). Small clusters of three markers were stuck on the subject's thumbnail and fingernail. Each cluster was connected to the Optotrak system by a single, flexible wire that was taped to the subject's arm. Subjects declared that the clusters and the wires did not bother them. The data of the three markers of a cluster were used to calculate the thumb and index finger's position off line. We wanted to evaluate the location of the point at which the digit contacted the object instead of the position of the cluster attached to the nail. In order to do so, a one-second recording was taken of an additional marker held in a precision grip. The position of this additional marker was regarded as the thumb or finger's position, and its position relative to the two clusters during this additional recording was used to calculate the relevant position in the subsequent analysis. Three markers were attached to the experimental cube.

Data analysis

We determined the tangential velocity of each digit by numerical differentiation. For each digit, movement onset was defined as the moment in time at which the digit had moved $5 \mathrm{~mm}$ from its starting position. We determined the endpoints of the digits' movements using the Multiple Sources of Information Method (Schot et al. 2010). This method determines the most likely endpoint by combining various parameters. In our case, the end was when the digits moved slowly, and were close to the cube, but the cube had not moved. The precise measure we used was:

$$
\max _{v_{\text {cube }}(t)=0}\left(1-\frac{v(t)}{v_{\max }}\right)\left(1-\frac{d(t)}{10}\right)
$$

in which $d(t)$ is the distance between the digit and the centre of the cube's surface in $\mathrm{cm}, v(t)$ is the velocity of the digit, and $v_{\max }$ is the maximum velocity of that digit. Movement time (MT) was calculated as the difference in time between movement onset and endpoint.

In addition to parameters that describe the digits' movements, we want to compare parameters of grasping (such as peak grip aperture) with equivalent measures during single-digit tasks. In the latter case, the parameters in a single trial are not equivalent to the ones in grasping (e.g. the grip aperture is meaningless in a finger push trial), but we can combine movements of the active digits across trials. Thus, we can define a measure for the push task that is equivalent to the grip aperture by taking the distance between the finger in a finger push trial and the thumb in a thumb push trial. We used the average trajectories of the active digits to do so. In order to make a fair comparison, we also calculated the parameters in the grasping trials based on the digits' average trajectories.

The trajectories were divided into one hundred segments of equal length. The length was defined as the sum of the horizontal distances between consecutive samples. The segment ends were determined using linear spatial interpolation. The relative time (time relative to movement onset divided by the movement time) and the threedimensional position were determined for the 101 ends of the segments. These data were used to calculate average movement trajectories.

We compared movement parameters (movement time, maximum velocity) between conditions using $t$-tests, using an alpha-level of 0.05 . These tests were paired $t$-tests if they compared the two digits in grasping, and unpaired $t$-tests for all other comparisons. We furthermore used an ANOVA to test whether the condition 
affected the within-subject variability in the above-mentioned parameters.

In order to evaluate how much the digits deviated from each other's mirror image, we defined a measure for this finger-thumb difference by averaging the two paths of the active digits for each of the 101 ends of the segments. For grasping, the resulting trajectory corresponds to the transport component. For digits' paths that are each other's mirror image, the resulting trajectory will be a straight line. We will refer to the deviation from a straight line as the finger-thumb difference.

In our initial paper on the control of digits during grasping, we made quantitative predictions using a minimum-jerk model (for details, see Smeets and Brenner 1999). In this model, we implemented independent control of the digits by letting each digit move as smoothly as possible from the start to the final position, with constraints on the velocity and acceleration at both movement onset and contact. We assumed that the two digits started together with zero velocity and acceleration. Contact also occurred simultaneously, with zero velocity and a final deceleration that was perpendicular to the surface.

\section{Results}

On average, subjects took $710 \pm 134 \mathrm{~ms}$ (mean $\pm \mathrm{SD}$ ) to complete the movements. Pushing was faster than touching $(652 \pm 121 \mathrm{~ms}$ and $771 \pm 122 \mathrm{~ms}$, respectively; $P<$ $0.001)$. The movement times for grasping (702 $\pm 138 \mathrm{~ms})$ did not differ from either of these values. With respect to temporal constraints, grasping is therefore intermediate between our two one-digit tasks. Movements in which the thumb was the active digit were slightly (42 ms, not significant) faster than ones in which the index finger was the active digit. Also in grasping, the movement time did not differ between the two digits. The maximum velocity of the digits was about $0.45 \mathrm{~m} / \mathrm{s}$. In all tasks, the maximum velocity of the index finger was higher than that of the thumb (difference $0.04 \mathrm{~m} / \mathrm{s}, P<0.001$ ). The maximum velocities did not differ significantly between the three tasks. Neither did the within-subject standard deviations of any of these measures.

Figure 2 shows a top view of all movement paths of subject S6 for three of the five conditions. The movements of the digits are quite reproducible, except for the passive thumb when pushing with the finger (the continuous magenta lines). The movements of the digits are different when passive than when active: the paths of the passive digit do not curve towards the object's surface. For this subject, the paths of the active digits are more or less the same when pushing, touching and grasping. We will concentrate on the question whether the movements of the active digits differ systematically between the tasks, and if so, on the nature of the difference.

Figure 3 shows the average paths of the active digits in all tasks for all subjects. Subject S6 whose data were shown in Fig. 2 is a subject for whom the three tasks yield almost identical paths. For most other subjects, the digits stay closer to each other when grasping than one would predict from the paths in the other two tasks. It is clear from this graph that subjects have their own characteristic movement pattern. Some subjects curve leftward (towards the thumb, e.g. S1), others rightward (e.g. S2) in all tasks. According to the minimum-jerk model, the vertical component should be a straight line, which it definitely is not (right panels of Fig. 3).

As anticipated on the basis of the literature, the paths of the finger and the thumb were clearly not each other's mirror image when grasping. The difference is not simply that the paths of the index fingers are less straight than those of the thumbs: subjects seem to have their own preferred curvatures for both finger and thumb. To quantify the differences between the digits in grasping, and to test

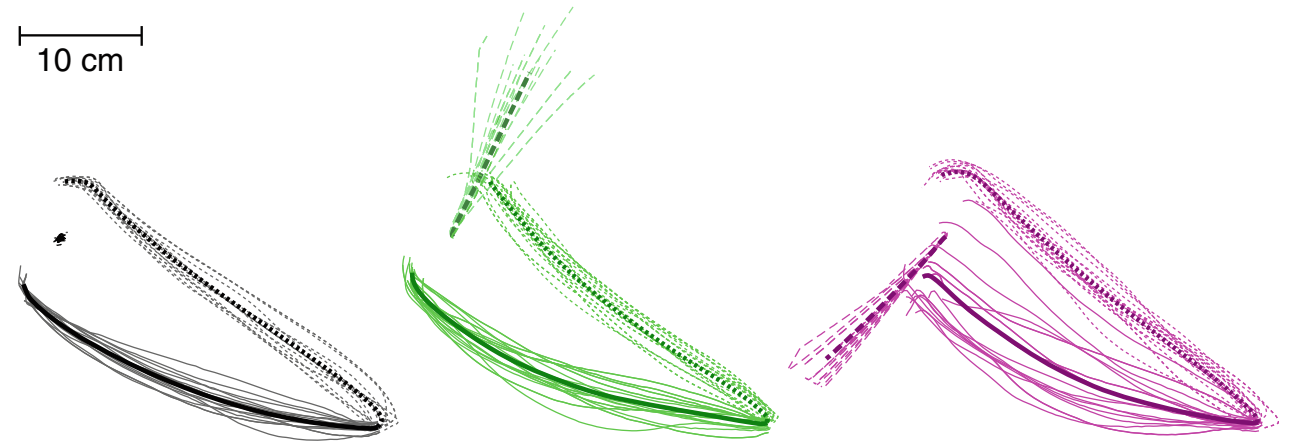

Fig. 2 Example trajectories. Top view of subject S6's movement paths. Thin curves represent single trials; dark thick curves represent average paths. The movements of thumb (continuous lines), index finger (dotted lines) and cube (dashed lines) are indicated for grasping the object (left panel, grey curves), and for pushing the object with the thumb (green curves, central panel) and index finger (magenta curves, right panel) 


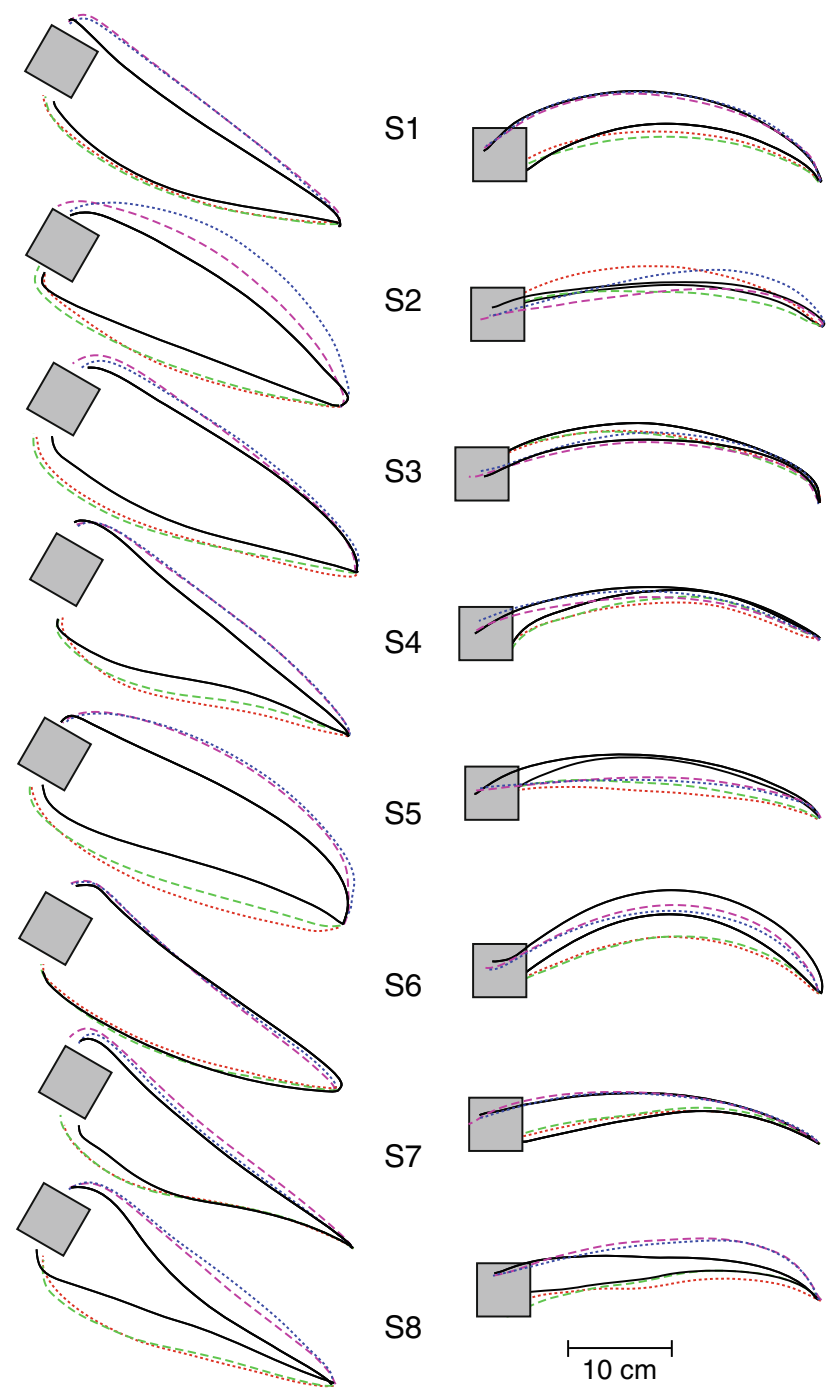

Fig. 3 Overview of the active digits' paths when performing the three tasks. A top view (left column) and a side view (right column) is given for each subject (S1-S8). The colours and styles of the curves indicate the task: continuous black: grasping; dotted red: touch with thumb; dotted blue: touch with finger; dashed green: push with thumb; dashed magenta: push with finger

whether the same differences between the two digits also exist when performing the single-digit tasks, we determined the path of the average of the two digits (see Fig. 4). If the digits' movements were each other's mirror image, as predicted by the minimum-jerk model, this analysis would always give a straight line (dashed line). There are clear deviations from a straight line. The differences between the digits differ between subjects (different colours in left column graphs), but are rather consistent across tasks (different line styles in central and right column graphs). This means that the difference between the paths of the two digits is due to specific properties of the individual digits' movements and not due to specific properties of the control for grasping.
As discussed earlier, it is clear from the trajectories in Fig. 3 that subjects' individual digits moved further from a straight path in the single-digit tasks than in grasping. This is summarised in Fig. 5a. The peak distance between the active digits in the single-digit tasks is clearly larger than in grasping and occurs earlier (paired t-test; $P<0.005$ ). Such a negative correlation between the peak grip aperture and its timing is predicted by the minimum-jerk model for grasping. It occurs when the single free parameter of that model is varied (Smeets and Brenner 1999). For each subject in each task, we therefore determined how much the hand opened wider than the final grip (when touching the object) and where (in terms of percentage of distance; Fig. 5b) and when (in terms of a percentage of time; Fig. 5c) this happened. In both cases, we find a very good correlation $\left(r^{2}=0.84\right.$ for position and $r^{2}=0.74$ for time) with a slope of $-4.9 \% / \mathrm{cm}$ for position and $-4.6 \% / \mathrm{cm}$ for time. Neither the slopes nor the intercepts differed between the three tasks. This linear relationship between the timing of peak grip aperture and the aperture itself is inconsistent with the non-linear prediction of the minimum-jerk model.

The standard deviation in the horizontal placing of the active digits on the cube's surface is about $7 \mathrm{~mm}$, irrespective of the task (Fig. 6). The standard deviation for the passive digits (in the same direction and at the same time) is twice as large (14 mm), again independent of the task.

\section{Discussion}

Our main overall result is that several aspects of the movements of the digits in grasping were very similar to those in pushing and touching. This was so for the path of the average of finger and thumb (first prediction, Fig. 4), for the relationship between peak grip aperture and its timing (second prediction, Fig. 5b,c) and for the variability (third prediction, Fig. 6). These results can be regarded as clear support for our hypothesis that the movements of the digits themselves are controlled in grasping, not the opening of the grip (Smeets and Brenner 1999). The fact that the grip aperture was smaller than could be expected from the movements in the single-digit tasks (Fig. 5a) shows that the movements of the digits in grasping are not completely independent. This difference might be caused by the elastic tissue of the hand pulling the digits towards each other. Such elastic tissue would exert equal forces in opposite directions, and thus leave the average path unaffected. This is in line with the results of Fig. 4, showing similar average paths for all tasks.

It has been argued before that the thumb and finger have different roles in grasping: that the grip is made by moving the index finger relative to the thumb, which is regarded as the stable transport component (Wing and Fraser 1983; 

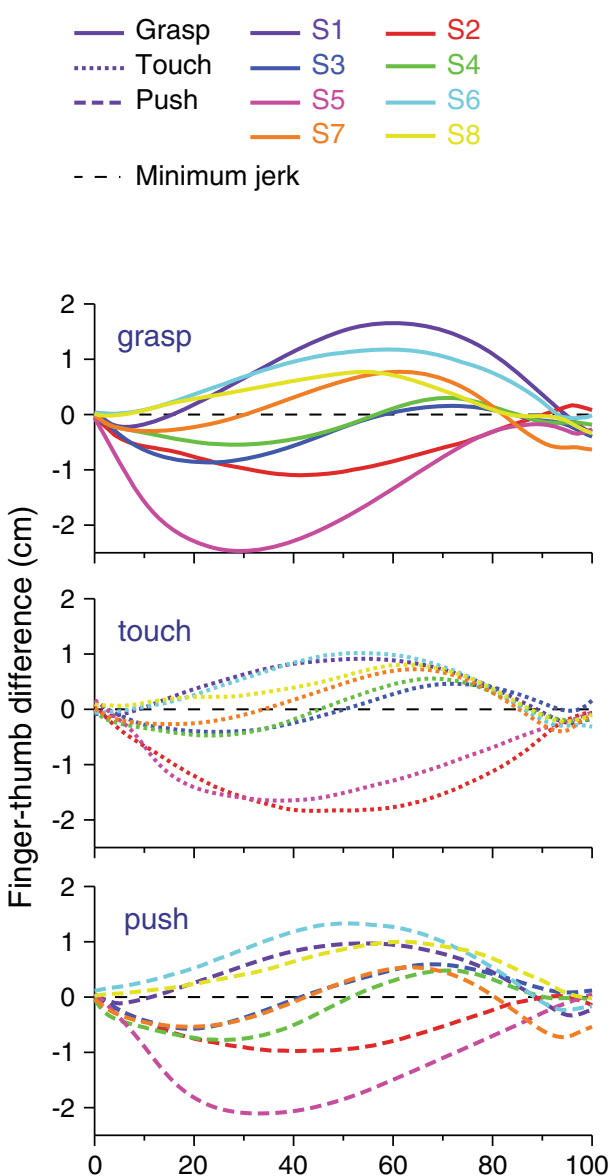
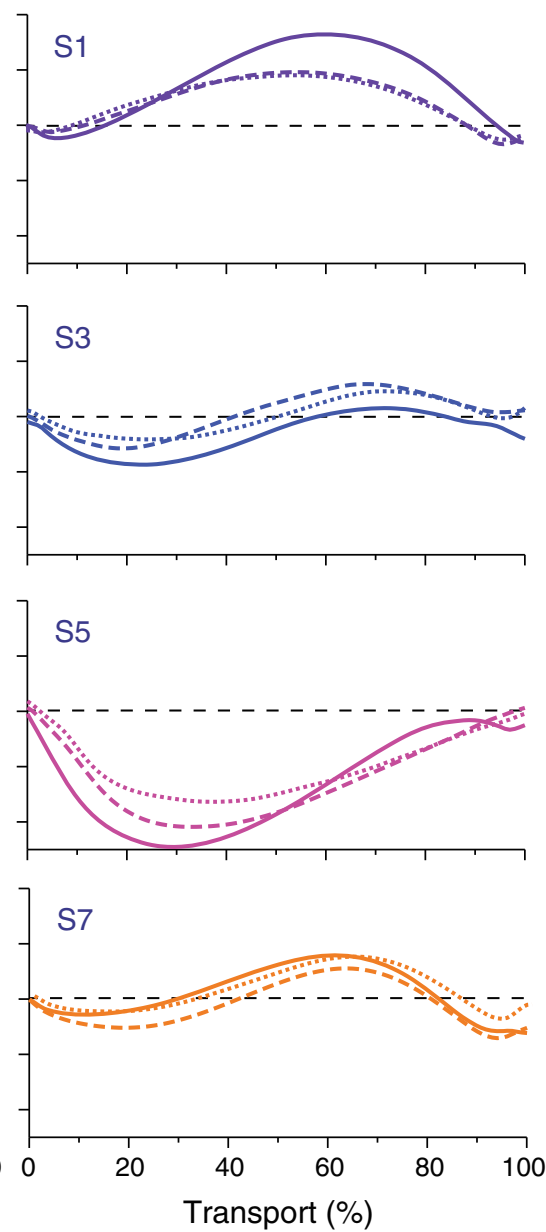

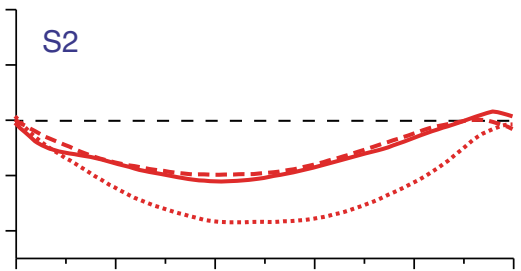

S4
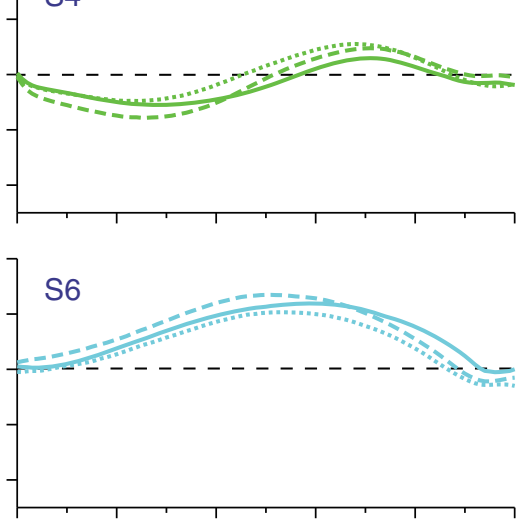

s8

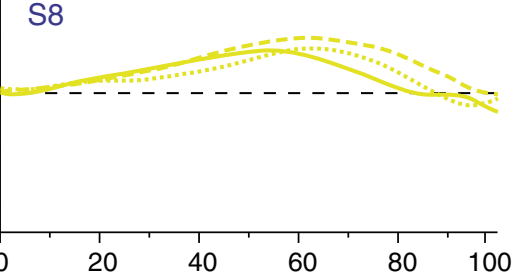

Fig. 4 Finger-thumb differences in the digits paths. The fingerthumb difference (the lateral deviation in the average of the active finger and thumb) is plotted as a function of the percentage of the movement to the cube. For grasping, the finger-thumb difference is equivalent to the lateral deviation of the transport component. If the thumb curves farther out than the finger, this measure will be positive
Haggard and Wing 1997). In the present study, the difference in path curvature between the digits is not systematic across subjects: for some subjects the finger deviates more than the thumb, but for others it is the other way around (Fig. 3), leading to subject-specific fingerthumb differences (Fig. 4). The precision is also the same for both digits, independent of whether they were active or not (Fig. 6). More importantly, the same differences between the digits were visible in the single-joint tasks. Apparently, subjects deal with the specific properties (thickness, segment length, etc.) of their finger and thumb in a certain way, independent of whether they are used in grasping or in single-digit pushing or touching.

A recent attempt to falsify the independent digit hypothesis was performed by van de Kamp and Zaal (2007). Subjects grasped an object that could change in such a way that only the target position of one of the digits was perturbed. In some cases, they found that the movement of the other digit changed to some extent; and in other cases, they found no significant effect. Why the digit for which the target did not change was affected in some cases is not clear, as simple kinematic parameters were not affected. The authors correctly claimed that any cross-talk is in conflict with purely independent control. We already showed in earlier experiments that the independence of the digits was not complete: there is a small but clear correlation between the digits (Smeets and Brenner 2001). This correlation was absent in bimanual grasping (a task that is similarly organised as unimanual grasping; Tresilian and Stelmach 1997), suggesting that the correlation is caused by anatomical factors rather than a control strategy. Such an anatomical factor could be the elastic tissue connecting the finger and thumb. Elastic tissue connecting the finger and thumb, combined with independent control of the digits, does not only explain the correlation between the digits (as reported by Smeets and Brenner 2001), but also the smaller grip aperture in grasping than one would expect on the basis of the single-digit tasks (as we found in the present study).

In our original proposal, we modelled the independent control of the digits with a minimum-jerk model. The 
Fig. 5 Grip aperture in the three tasks. In all tasks, grip aperture is defined as the distance between the average path of the active index finger and active thumb at corresponding positions on their paths. For touching and pushing, such apertures obviously never occurred in a single trial. a The distance between the digits as a function of time. $\mathbf{b}, \mathbf{c}$ The relation between peak grip aperture and the position (B) or time (C) of its occurrence. Different tasks are indicated by different symbols; different subjects by different colours (colour coding as in Fig. 4). Continuous line: straight line fit to the data of all tasks; dashed curve: minimumjerk prediction (no fit parameters)

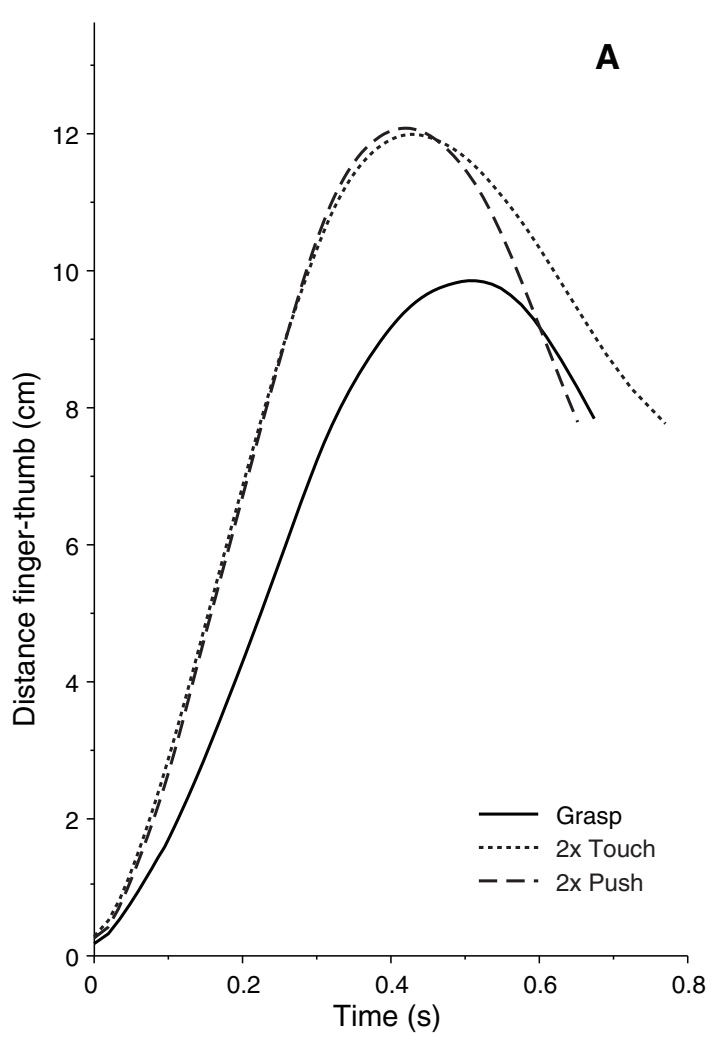

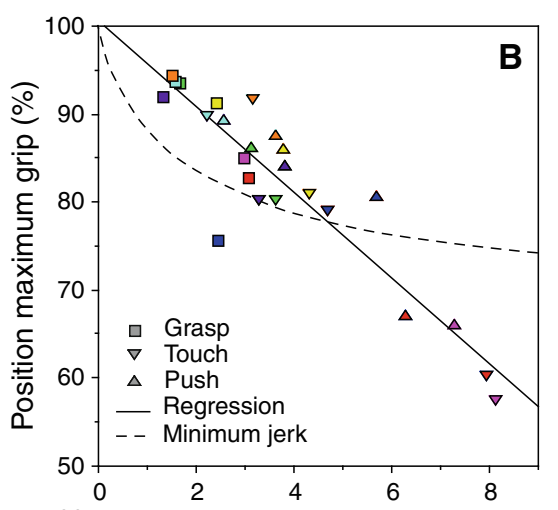

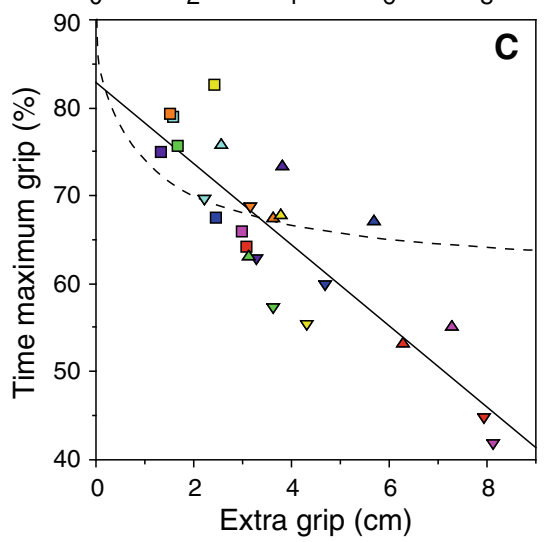

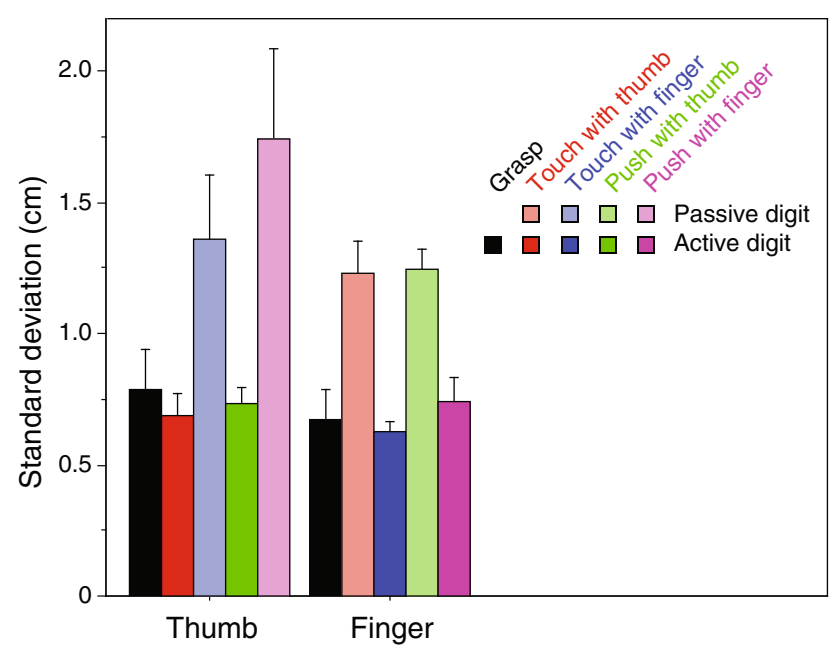

Fig. 6 Variability in the digits' end positions in the five tasks. The standard deviation parallel to the cube's surface (the square root of the sum of the variances in the forward and vertical direction). The variability in the active digit's position is the same for the two digits in the three tasks and is much smaller than that of the passive digit in touching and pointing. Error bars indicate the standard error of the mean (across subjects)

present study shows that although this might yield a good description of average behaviour, it clearly misses some details. Examples in the present study are the linear relationship between peak grip aperture and its timing (the model predicts a non-linear relation: Fig. 5) and the subject-specific finger-thumb differences (Fig. 4). Note that our original model inspired us to look for the correlation between peak grip aperture and its position in the movement. The very systematic relationship we find differs from the one we predicted (Fig. 5) and is therefore a good test for other candidate models for grasping.

The data deviate more substantially from the minimumjerk model in the vertical component of the movement. According to the model, the digits should move in a straight line (glide across the table), which they clearly did not (right column of Fig. 3). This deviation is not specific to grasping, but a general aspect in all our tasks. It is therefore in line with the independent digit control hypothesis. The large deviation from a straight movement in the vertical direction could be regarded as an argument against the minimum-jerk model, or more generally, against planning movements in extrinsic space (and in favour of joint-based planning, Desmurget et al. 1998; Desmurget et al. 1999). An alternative view is to regard the vertical component as the consequence of an additional constraint (the table as an obstacle to avoid) that might be incorporated in the spatial planning. This latter view would also give a possible explanation for the differences between conditions in the vertical component. Most subjects held their digits that were not used (little, ring and middle finger) in a different posture during grasping than during the other two tasks. In order to avoid contact with the table, they should therefore lift the hand differently. 
An important aspect of our model is that it is derived from task constraints, rather than anatomical constraints. The idea behind this is that our movements are organised in such a way that we can move without the anatomical constraints interfering with the execution. How this is done is not made explicit, but movements of distal joints will have to compensate for limitations of more proximal joints and vice versa. It is therefore not surprising that when expressed in a coordinate system that is attached to the hand (neglecting the contributions of proximal joints), the movements of the digits deviate clearly from a minimumjerk trajectory (Kamper et al. 2003). It is promising for our view on grasping that the same laboratory has reported a similar pattern of digit movements in pointing as in grasping (Cruz and Kamper 2006). The minimum-jerk model can describe independent control of the digits in grasping, pointing and touching. Although this model cannot describe all details, it has the advantage of being very simple (Smeets and Brenner 2002; Smeets et al. 2009).

It is clear that a simple model based on independent control of the digits (such as presented in Smeets and Brenner 1999) would not have predicted all the current results. We have argued above that some interaction between the digits needs to be incorporated in such a model to explain the differences between the individual digits' paths when grasping and when performing the other tasks. Does this imply that models based on the classical distinction between transport and grip yield a better description of the data? We do not think so, as no implementation of the classical model would have predicted our main finding: a striking correspondence in the difference between the digits' paths when grasping and when performing the two singledigit tasks (Fig. 4). Moreover, incorporating an interaction between the individual digits does not make the model more complex than the classical description using the variables transport and grip, because that description also requires an interaction between its two independent variables (Paulignan et al. 1990). We therefore conclude that our experimental results support the idea that grasping should be regarded as consisting of controlling the digits, rather than transporting the hand and shaping the grip.

Open Access This article is distributed under the terms of the Creative Commons Attribution Noncommercial License which permits any noncommercial use, distribution, and reproduction in any medium, provided the original author(s) and source are credited.

\section{References}

Bennis N, Roby-Brami A (2002) Coupling between reaching movement direction and hand orientation for grasping. Brain Res 952:257-267
Biegstraaten M, Smeets JBJ, Brenner E (2003) The influence of obstacles on the speed of grasping. Exp Brain Res 149:530-534

Cruz EG, Kamper DG (2006) Kinematics of point-to-point finger movements. Exp Brain Res 174:29-34

Desmurget M, Pelisson D, Rossetti Y, Prablanc C (1998) From eye to hand: planning goal-directed movements. Neurosci Biobehav Rev 22:761-788

Desmurget M, Prablanc C, Jordan M, Jeannerod M (1999) Are reaching movements planned to be straight and invariant in the extrinsic space? Kinematic comparison between compliant and unconstrained motions. Q J Exp Psychol A 52:981-1020

Flash T, Hogan N (1985) The coordination of arm movements: an experimentally confirmed mathematical model. J Neurosci 5:1688-1703

Franz VH, Hesse C, Kollath S (2009) Visual illusions, delayed grasping, and memory: no shift from dorsal to ventral control. Neuropsychologia 47:1518-1531

Ganel T, Tanzer M, Goodale MA (2008) A double dissociation between action and perception in the context of visual illusions-opposite effects of real and illusory size. Psychol Sci 19:221-225

Haggard P, Wing A (1997) On the hand transport component of prehensile movements. J Mot Behav 29:282-287

Kamper DG, Cruz EG, Siegel MP (2003) Stereotypical fingertip trajectories during grasp. J Neurophys 90:3702-3710

Kleinholdermann U, Brenner E, Franz VH, Smeets JBJ (2007) Grasping trapezoidal objects. Exp Brain Res 180:415-420

Mon-Williams M, McIntosh RD (2000) A test between two hypotheses and a possible third way for the control of prehension. Exp Brain Res 134:268-273

Paulignan Y, MacKenzie C, Marteniuk R, Jeannerod M (1990) The coupling of arm and finger movements during prehension. Exp Brain Res 79:431-435

Paulignan Y, Frak VG, Toni I, Jeannerod M (1997) Influence of object position and size on human prehension movements. Exp Brain Res 114:226-234

Schot WD, Brenner E, Smeets JBJ (2010) Movement endpoints can be determined robustly by combining multiple sources of information. J Neurosci Methods 187:147-155

Smeets JBJ, Brenner E (1999) A new view on grasping. Mot Control 3:237-271

Smeets JBJ, Brenner E (2001) Independent movements of the digits in grasping. Exp Brain Res 139:92-100

Smeets JBJ, Brenner E (2002) Does a complex model help to understand grasping? Exp Brain Res 144:132-135

Smeets JBJ, Brenner E, Biegstraaten M (2002a) Independent control of the digits predicts an apparent hierarchy of visuomotor channels in grasping. Behav Brain Res 136:427-432

Smeets JBJ, Brenner E, de Grave DDJ, Cuijpers RH (2002b) Illusions in action: consequences of inconsistent processing of spatial attributes. Exp Brain Res 147:135-144

Smeets JBJ, Glover S, Brenner E (2003) Modeling the timedependent effect of the Ebbinghaus illusion on grasping. Spat Vis $16: 311-324$

Smeets JBJ, Brenner E, Martin J (2009) Grasping occam's razor. In: Sternad D (ed) Progress in motor control: a multidisciplinary perspective, vol 629. Springer, Berlin, pp 499-522

Tresilian JR, Stelmach GE (1997) Common organization for unimanual and bimanual reach-to-grasp tasks. Exp Brain Res 115:283-299

van de Kamp C, Zaal FTJM (2007) Prehension is really reaching and grasping. Exp Brain Res 182:27-34

Wing AM, Fraser C (1983) The contribution of the thumb to reaching movements. Q J Exp Psychol A 35:297-309 